\title{
The Critical Role of the Atmosphere in Dip-Coating
}

\section{Process}

\author{
Elisa Bindini ${ }^{1}$, Guillaume Naudin ${ }^{1}$, Marco Faustini ${ }^{1}$, David Grosso ${ }^{2}$, and Cédric Boissière ${ }^{1 *}$
}

1. Laboratoire Chimie de la Matière Condensée de Paris, UMR UPMC-CNRS 7574, Université

Pierre et Marie Curie (Paris 6), 4 Place Jussieu 75252 Paris, France

2. IM2NP - Case 142 Avenue Escadrille Normandie Niemen 13397 Marseille (France)

\begin{abstract}
Dip-coating is a common liquid deposition technique employed in research, but also for industrial production, to obtain polymer, hybrid and inorganic thin layers of controlled thickness. During liquid deposition, the substrate withdrawal speed allows in principle an easy tuning of deposited film thickness (first modeled by Landau and Levich). Yet, experimentally, unexplained thickness irreproducibility or strong fluctuations of the sol-gel films are often observed when coating large substrates, which is a critical issue for optical coatings such as antireflective/reflective coatings. In this study, we pointed out for the first time that uncontrolled solvent relative pressure gradients (coming from solvent evaporation) are responsible for these 1
\end{abstract}


thickness fluctuation issues. We investigated and quantified their impact for various solutions (of sol-gel or polymer), and pointed out that the solvent evaporation rate is not constant but strongly depends on the geometric configuration of the dip-coating experiment. From this understanding, we demonstrated how an accurate tuning of processing atmosphere can provide a very good control on layer thickness in the practical case of the deposition of anti-reflective water repellent coating. In a second example, we used this phenomenon for developing a very easy synthesis strategy leading to giant and controlled thickness gradient profiles. 


\section{INTRODUCTION}

Dip-coating from solution is a widely used method to deposit thin films on various substrates. With this liquid coating process, a homogeneous layer is easily spread on the substrate surface and the solvent evaporation leads to the formation of a solid film with a good control on structure and thickness ${ }^{1-4}$. In principle, both organic and inorganic thin layers can be obtained by this method, and studied for many applications ranging from electronics ${ }^{5-8}$, optical coatings $^{9}$, photocatalysis ${ }^{10,11}$, and more ${ }^{12-14}$. Despite the wide use of sol-gel coatings both in industrial production and in research laboratories, the physical-chemical phenomena taking place during the dip-coating process are still not fully understood. The first definition of draining and dragging phenomena involved in dip-coating was published by Landau and Levich (LL) in 1942 for polymers ${ }^{15}$, who built up a theoretical model to describe the film formation mechanism and predicted the final thickness in certain conditions. We will refer to this regime, which follows the LL model, as "Draining regime". Since then, dip-coating processing has been used for depositing thin films from very different solutions which not always satisfy hypotheses of LL model. For example, whenever solvent evaporation is taking place, several critical parameters may change with time (like rheological properties and surface tension), due to the increase of concentration of non-evaporating components. The consequence of this is that a new low dragging speed deposition regime (so called Capillarity Regime - CR), not described by LL model, exists for this type of systems, as recently reported $^{16}$ (Figure 1).

Solvent evaporation affects significantly the deposition process, its rate becoming a critical parameter that radically influences the deposited film thickness. The Capillarity Regime allows obtaining thick films with highly diluted solutions at ultralow withdrawal speeds and can be used when species to deposit cannot be dissolved or dispersed in high concentrations. 
Moreover, in the CR, a solvent with high surface tension, such as water, can be used without dewetting problems that are always encountered in the draining regime. For sol-gel solutions, many progresses have been made to better understand the global dynamics related with dipcoating deposition, in order to reach a better control on final film thickness and structuration $^{16-19}$. A global, semi-empirical model giving a satisfactory description of dipcoating process, covering a broad withdrawal speed range (from 0.001 to $10 \mathrm{~mm} . \mathrm{s}^{-1}$ ) was published by Faustini et al. ${ }^{16}$; it combines in a single equation both contributions of Capillarity and Draining effects (Equation 1).

At high withdrawal speed, the amount of solvent evaporated at the liquid meniscus is negligible, thus the film formation follows the LL model. At ultralow withdrawal speeds, solvent evaporation takes place at the liquid meniscus, which is continuously fed through capillary rise from the solution. In this case, no dragging is involved and the film thickness is directly related to the evaporation rate $E$. At intermediate speeds, both contributions are balanced. The speed range of this intermediate domain strongly depends on both solution composition and processing conditions such as temperature. The equation 1 predicts the final film thickness across the full span of withdrawal speeds, taking into account both the capillarity and the draining regime behaviours:

$$
h_{0}=k_{i}\left(\frac{E}{L} \frac{1}{u}+D u^{2 / 3}\right)
$$

where $k_{i}$ and $D$ are constants characteristic of the solution, $L$ is the width of the deposited layer and $u$ is the withdrawal speed.

This semi-empirical model describes satisfactorily the impact of withdrawal speed onto film thickness assuming that the processing conditions do not change during coating. Often, when relatively large surfaces have to be covered, one needs to use an adapted reservoir to prevent the use of a large amount of solution. In such cases, conditions may not be identical all over the substrate surface and thickness non uniformity is often noticed. The variation of 4 
processing conditions is mainly related to the variation of the level of the solution with respect to the reservoir top limit during withdrawal, which changes the evaporation conditions along the substrate position due to vapor confinement. As an example, in Figure 2 is shown a glass substrate coated with an anti-reflecting layer made by dip-coating process at constant temperature and withdrawal speed in the conditions illustrated in Figure $2 \mathrm{~b}$. The transmission spectra point out that, along the sample, the intensity of transmitted light changes, which means that the anti-reflective layer thickness is not uniform from the top to the bottom of the sample. By measuring the film thickness at several heights, we evidenced that the coating layer becomes thinner with the distance of the solution level to the top edge increasing, confirming that the geometry, dimensions and filling level of the reservoir used for dipcoating do have an strong impact on the amount of material deposited, causing thus very significant thickness non homogeneity. Other evidences of such an effect are reported in Figure 3 where the variations of thickness with respect to the withdrawal rate are plotted for a $\mathrm{TiO}_{2}$ sol-gel solution prepared in similar conditions but with different reservoir geometries or filling levels. Because the reservoir geometry and filling level mainly influence the local vapor pressure governing the evaporation rate, we investigated the influence of solvent relative vapor pressure in the atmosphere on the final thickness with respect to the withdrawal speeds, which is a parameter usually not taken into account.

It is known that the relative solvent vapor pressure during film formation can impact the material structure ${ }^{20,21}$ but until now, the effect of a solvent enriched atmosphere on the amount of material deposited was never extensively investigated. The purpose of this work is having an overview of the phenomenon and its mechanisms to be able to better handle and control the dip-coating process from small to large production scale.

The solvent vapor pressure being the main factor responsible for the behavior shown in Figure 3, several chemical systems were investigated to obtain dense $\mathrm{TiO}_{2}$ or mesoporous 5 
$\mathrm{SiO}_{2}$ films from ethanol-based sol-gel solutions, porous $\mathrm{SiO}_{2}$ films from water-based colloidal solution, and PS-PEO copolymer films from THF solution. All solutions exhibit a similar behavior, confirming the role of solvent relative vapor pressure in the atmosphere during dipcoating. We carried out an experimental investigation which demonstrates the importance of solvent relative vapor pressure not only in the ultra-low speeds regime, in which the evaporation drives the film formation process, but also at speeds usually considered as pure Landau-Levich regime, suggesting that the influence of capillarity regime can be relevant until relatively high speeds, depending on the solvent used.

\section{EXPERIMENTAL SECTION}

2.1 Materials. Absolute ethanol and Tetrahydrofuran were purchased from Normapure, $\mathrm{HCl}$ from Merck Millipore and $\mathrm{TiCl}_{4}, \mathrm{CTAB}$ (hexadecyltrimethylammonium bromide) and TEOS (tetraethylorthosilicate) were purchased from Aldrich and used without further purification. PS-b-PEO (polystyrene-b-polyethyleneoxide $\mathrm{Mn}=16400 \mathrm{Mn}=36400 \mathrm{Mw} / \mathrm{Mn}: 1.06$ ) was purchased from Polymer Source.

Ludox silica nanoparticles suspension, named HS40 was purchased from Aldrich. The original stock of colloidal suspension was diluted 10 times with water.

2.2 Solutions preparation. The anti-reflective coatings were made of mesoporous methylated $\mathrm{SiO}_{2}$ by dip-coating at $0.3 \mathrm{~mm} \mathrm{~s}^{-1}$ in dry air on sodalime glass. The solution composition is $0.5 / 0.5 / 5 / 40 / 0.006 / 0.006$ TEOS/MTEOS $/ \mathrm{H}_{2} \mathrm{O} / \mathrm{EtOH} / \mathrm{HCl} / \mathrm{F}-127$ for the samples shown in Figure 6 (a-c) and 0.5/0.5/4/20/0.007/0.006 for the sample shown in Figure 6 (d). After the deposition, samples were cured at $450^{\circ} \mathrm{C}$ for 10 minutes. Both thickness measurements and transmission spectra were measured by spectroscopic ellipsometry.

The $\mathrm{TiO}_{2}$ solution was prepared with a molar ratio of 1:55:10 $\mathrm{TiCl}_{4} / \mathrm{EtOH} / \mathrm{H}_{2} \mathrm{O}$ and used fresh. The experimental investigation was performed using dense $\mathrm{TiO}_{2}$ as a model system 
since the film formation mechanisms are well known and titania thin films are widely employed for many applications ${ }^{22-24}$. The final films obtained were cured at low temperature $\left(130{ }^{\circ} \mathrm{C}\right)$ to stabilize them keeping an amorphous structure, in order to avoid uncontrolled crystallization followed by $>50 \mathrm{~nm}$ surface roughness, not suited for ellipsometric analysis ${ }^{25}$. $\mathrm{All} \mathrm{TiO}_{2}$ films analyzed had a refractive index at $700 \mathrm{~nm}$ between 1.985 and 2.020.

The thickness values were obtained by using a regular beam $(3 \mathrm{~mm})$ that allows averaging the thickness fluctuation (typically 7\%) due to stick and slip phenomena in the capillarity regime.

The mesoporous $\mathrm{SiO}_{2}$ solution was prepared using tetraethyl orthosilicate (TEOS) as inorganic precursor and hexadecyltrimethylammonium bromide (CTAB) as template surfactant. TEOS and CTAB are added to EtOH and in the end is added aqueous acidic solution ( $\mathrm{pH}=2$ by $\mathrm{HCl}$ addition), the molar ratio used is $1 / 0.14 / 40 / 5$ TEOS/CTAB/EtOH/ $\mathrm{H}_{2} \mathrm{O}$. The solution is stirred at room temperature for $72 \mathrm{~h}$ before use.

The PS-b-PEO polymer solution was prepared in a concentration of $3.33 \mathrm{~g} / \mathrm{L}$ using THF as solvent.

2.3 Processing. Dip-coating was performed using an ACEdip equipment (from SolGelWay, Paris, France) in an atmosphere of dry air $(\mathrm{RH}<5 \%$, flux $2.5 \mathrm{~L} / \mathrm{min})$ for the $\mathrm{TiO}_{2}$ experiments with different levels of solution in the reservoir and injecting EtOH-saturated air for tests at different vapor pressures, as previously explained. Air was bubbled into $\mathrm{EtOH}$ at $50{ }^{\circ} \mathrm{C}$ to be saturated with the solvent vapors and then passed through $\mathrm{EtOH}$ at $25{ }^{\circ} \mathrm{C}$ before entering in the dip-coater chamber. In this way, the excess of EtOH vapors recondenses from hot air in the solvent at $25^{\circ} \mathrm{C}$, avoiding the condensation in the wires and inside the dip-coater, which would cause strong irreproducibility. Then the EtOH enriched air is mixed with dry air, in proportions managed by a mass flow controller, and injected in the dip-coater. A total air flux of $2.5 \mathrm{~L} / \mathrm{min}$ is maintained during the deposition process, otherwise, especially for slow 7 
withdrawal speeds, the ethanol relative vapor pressure in the atmosphere changes too fast, leading to irreproducible results, since the chamber is not a hermetically close system but communicates with the external environment. For the other systems tested no flux of air was used during the dip-coating.

The temperature in the chamber was $40{ }^{\circ} \mathrm{C}$ for the solution of $\mathrm{SiO}_{2}$ nanoparticles in water and for the polymeric solution, $26^{\circ} \mathrm{C}$ in the other experiments.

Films were prepared on silicon substrates $(1 \times 7 \mathrm{~cm})$ cleaned with acetone and, after the coating, they were cured at $130{ }^{\circ} \mathrm{C}$ for 1 hour, except the polymeric and the colloidal ones, which have not been thermally treated. The $\mathrm{SiO}_{2}$ films reported in figure 7 have been stabilized at $130^{\circ} \mathrm{C}$ for one night and then cured at $450^{\circ} \mathrm{C}$ for 10 minutes.

2.4 Characterization. The refractive index and the thickness of final films were measured, after the stabilizing thermal treatment, by spectroscopic ellipsometry performed on a UV-IR (193-1690 nm) variable angle spectroscopic ellipsometer (VASE) M2000DI from Woollam at an incidence angle of $70^{\circ}$, and the data analysis was performed with the CompleteEASE software.

\section{RESULTS AND DISCUSSION}

In many industrial applications, for which it is important to control the thickness of the thin layer deposited, dip-coating is often the chosen method to perform a liquid deposition on a substrate $^{26}$. In a simple approach, one can notice that filling the container at various levels or using different containers could lead to very different thicknesses of the final film obtained, as evidenced qualitatively in Figure 3. As expected from the reference model ${ }^{16}$, films thickness decreases when withdrawal speed rises until it reaches a minimum after which it starts to increase again, following the Landau-Levich model.

8 
Yet, the curve of film thickness versus withdrawal speed shifts between different experiments, meaning that, for the same experimental conditions and the same speed, we obtain films with different thicknesses.

As evidenced from Figure 3, the gap in thickness is huge in the ultralow speeds regime and is progressively reduced with increasing speeds. The factor which is responsible for this behavior is likely to be the partial vapor pressure of the solvent in the atmosphere originating from the solution evaporating from the reservoir and from the deposited layer.

\subsection{Influence of solvent relative vapor pressure in the atmosphere}

To investigate this hypothesis, we performed a first set of dip-coating with an ethanol-based $\mathrm{TiO}_{2}$ sol-gel solution. The dip-coated atmosphere was progressively enriched with ethanol employing the gas injection system described in SI 6. Briefly, dry air was bubbled into hot ethanol at $50{ }^{\circ} \mathrm{C}$ to be saturated with the solvent vapors and then passed through cold ethanol at $25^{\circ} \mathrm{C}$ before entering in the dip-coater chamber. After the passage in ethanol at ambient temperature, the solvent-saturated air was mixed with dry air in different proportions, regulated by mass flow controllers, and injected within the dip-coater.

From the ratio between dry air and EtOH-enriched air, we infer the ethanol relative pressure injected into dip-coating chamber. The relative vapor pressure values reported in Figure 4 are values related to the air injected in the chamber, they are not the effective ethanol vapor pressure values of the atmosphere within the chamber. Probably full saturation in EtOH $(100 \%)$ is never reached, because the system is not perfectly sealed and exchanges air with the outside. All the experiments were performed with a reservoir filled up with solution up to the top. Plotting the thickness versus withdrawal speed by equation 1 , we can clearly see a shift of the point of minimum thickness towards lower withdrawal speed values with increasing solvent vapor pressure, as shown in Figure 4, going from a minimum thickness of $62 \mathrm{~nm}$ in dry air until $29 \mathrm{~nm}$ when injecting EtOH-saturated air. From the plots in Figure 4 we 9 
notice a linear correlation between minimum thickness and solvent partial vapor pressure increase.

As expected, the influence of higher vapor pressure atmosphere is stronger in the capillarity regime where the thickness of the deposited layer is directly proportional to the evaporation rate $E^{16}$ :

$$
h_{0}=\frac{c_{i} M_{i}}{\alpha_{i} \rho_{i}} \frac{E}{L} \frac{1}{u}=k_{i} \frac{E}{L} \frac{1}{u}
$$

Equation 2 gives the expression of final film thickness $h_{0}$ obtained in pure capillary regime versus withdrawal speed $u$. A more detailed description of the material proportion constant $k_{i}$ for the capillary regime includes the solution inorganic concentration $c_{i}$, the molar weight $M_{i}$ and the density $\rho_{i}$ of the stabilized material. $\alpha_{i}$ is the volume fraction of the inorganic material deposited in the final film and $L$ is the width of the deposited layer, which doesn't change during the dip-coating process. For most solutions, $k_{i}$ is constant and the plot of $h_{0} / k_{i}$ versus $1 / u$ plot gives a straight line with a slope corresponding to the solution depositing rate $E / L$, that is, the final thickness is proportional to the evaporation rate $E$. The evaporation process of a solution could be a complex phenomenon to discuss and is influenced by several parameters. In the most simple case for which no convection is involved, the evaporation rate $E$ of a solvent can be described following the Hertz-Knudsen equation (3) ${ }^{27,28}$ :

$$
E=A \alpha(K T)^{-1 / 2}\left(P_{(T)}^{*}-P_{a m b}\right)
$$

where $A$ is the evaporation surface area and $T$ is temperature, $\alpha$ is the sticking coefficient of a gas molecule onto the surface and $K$ is a constant including the molecular mass, the Boltzmann constant and a numeric prefactor. Both $\alpha$ and $K$ are determined by solvent characteristics. The Equation 3 shows that $E$ depends on the difference between the solvent 
saturation vapor pressure $P^{*}$ at the temperature of the solution and its relative vapor pressure in the gas phase $P_{a m b}$.

Including $E$ in Equation 2 we obtain:

$$
h_{0}=\Gamma A T^{-1 / 2}\left(P_{(T)}^{*}-P_{a m b}\right) \frac{1}{u}
$$

Where $\ulcorner$ gathers all the constant factors previously described. By increasing the solvent relative vapor pressure in the atmosphere $P_{a m b}$, the evaporation rate $E$ is reduced and, as is evident from equation 4 , the thickness of the deposited layer $h_{0}$ will be smaller. The surface of the interface $A$ which, in our case, is the surface of the liquid meniscus at the contact with the substrate, is also playing a role in the kinetics of evaporation. As a direct consequence, every parameter which can lead to a different shape of the meniscus as the reservoir geometry, the contact angle between the substrate and the solution, the distance of the sample from the reservoir's edge, will also influence the evaporation rate and, consequently, the film thickness.

Theoretically, in the draining regime, the relative vapor pressure doesn't affect the final thickness of the film obtained, as described by LL model (Equation 5):

$$
h_{0}=k_{i} D u^{2 / 3}
$$

In this regime, the system reaches quickly the equilibrium thickness, meaning that the variations in viscosity and surface tension, caused by the presence of solvent vapors in the atmosphere, are small and that the evaporation process is slower than the time needed for the layer deposition.

In the intermediate speed range where both regimes have a contribution, we can expect differences in the final thickness due to the influence of the capillarity regime. The effect of higher ethanol relative vapor pressure in the atmosphere can easily explain the behavior observed in real experimental conditions, as reported in Figure 3. 


\subsection{Effect of meniscus position}

However, to be reproducible, not every height of the solution inside the reservoir is equivalent: when the liquid meniscus is above the container, the evaporation speed is controlled by the relative solvent vapor pressure within the dip-coater.

As soon as the meniscus is positioned within the solution's container, the relative solvent vapor pressure increases following a gradient from the external edge towards the inside. In this situation we observe experimentally thinner films. In Figure 5 is plotted the thickness of a film deposited a constant speed by dip-coating, versus the position of the liquid level within the container $\Delta z$. The final film thickness $h$ is normalized on $h_{0}$ which is the thickness of a film deposited in the same conditions but with a completely full container (Level 0). Values of $h / h_{0}$ underline that a small variation in the solution height inside the reservoir leads to huge differences in the amount of material deposited, up to a decrease of $80-90 \%$ in capillarity regime.

It is evident that there is always a gap in the amount of material deposited on the substrate; one has to highlight that, this effect is visible even in Landau Levich regime, and can be very strong when a highly volatile solvent, such as THF, is used. This is exemplified with the polymeric system polystyrene-block-poly(ethylene oxide) in Figure 5, that reveals a thickness variation of $70-75 \%$ even at withdrawal speed $5 \mathrm{~mm} \cdot \mathrm{s}^{-1}$ that is usually considered in "pure" Landau Levich regime. So, in this case, even for a high withdrawal speed, the evaporation process is faster than the liquid layer deposition. Below a given $\Delta z$ value, lowering further the liquid level does not bring any additional thickness variation. We assume this is due to the fact that within the solution reservoir, the atmosphere is nearly saturated, thus that evaporation speed is minimum. In such an experimental situation, the $P_{a m b}$ parameter of equation (4) is not a constant anymore but becomes a function describing the local evolution of the relative solvent pressure above the solution meniscus (which is directly related to the solvent relative 12 
pressure gradiant appearing with solution level lowering). How steep the initial thickness decreases (in other words, how high $P_{a m b}$ value increases) with solution lowering depends experimentally on the solvent volatility, on the withdrawal speed, and on the reservoir dimensions and shape. It can be determined experimentally in figure 5 by assuming that $h / h_{O}$ is proportional to $P_{a m b}(\Delta z) / P_{a m b}(0)$.

The shape of meniscus is another important parameter that can play a role in the thickness differences observed at different positions of the film. We verified that the meniscus shape changes noticeably only in the first $0.1 \mathrm{~cm}$ (data reported in SI), that is when the meniscus passes from outside the reservoir to within. We can observe thickness variations duting the first 2-3 cm on the film (Fig. 5), meaning that the solvent vapor pressure variation is mainly responsible for the described behavior.

The observed phenomenon can be indeed a real problem when coating large substrates. In fact, if the volume of the immersed substrate is significant with respect to the volume of the container, dragging will cause an important variation in the liquid level and, as a consequence, a difference in thickness between the top and the bottom of the sample. A direct consequence is that the shape and volume of the container does affect the film thickness profile in the capillarity regime (an example is given in SI 5).

When applied to the real case of an anti-reflecting layer, for which thickness variation strongly impacts the transmittance of a window, we verified that the samples coated with the same solution but starting from a different position of the meniscus (at the reservoir's top edge level, and some $\mathrm{cm}$ inside the reservoir) shows smaller difference in thickness and transmission along the specimen, as evident in Figure 6. One can of course minimize this effect by using high withdrawal speeds. Nevertheless, industrial production favors waterbased solution which imposes low speed or high temperature deposition conditions, both entailing significant solvent evaporation contributions. 
From Figure 5, we can infer that, to gain reproducibility, it is better to keep the liquid level some $\mathrm{cm}$ lower, to be on the plateau of vapor pressure profile. In such a case, obtaining the targeted thickness (about $80 \mathrm{~nm}$ for this antireflective coating deposited on sodalime glass) implies to readapt the concentration of the solution (see Figure 6). Moreover, it is better to use a large reservoir with respect to the volume of the substrate, in this way the dragging will impact less the level of the solution within the container than in the case of a smaller reservoir. Of course, the use of big reservoirs means that huge a volume of solution has to be prepared, which is often not possible or too expensive. An efficient technical solution to this problem called "bi-phasic dip-coating" method was recently reported. ${ }^{29}$ Its principle consists in using a reservoir as large as needed with a small amount of coating solution deposited at the top of a large volume of non-miscible and denser liquid, which is an efficient remedy to the issue investigated here.

\subsection{Towards controlled gradients}

Indeed, the variation of the solvent relative vapor pressure in the atmosphere during the dipcoating process may cause huge thickness gradient on the deposited films. We investigated this phenomenon and proposed some solutions to compensate it when the thickness uniformity of the layer is required. Nevertheless, considering that in some processing conditions the thickness decrease can be as important as $90 \%$, it would be very interesting to exploit this effect to fabricate graded films with a controlled thickness profile. The huge potential of thin films exhibiting functionality gradients obtained from films thickness gradient is well illustrated in the work of Faustini et al. ${ }^{30}$ In this study, thickness gradient were produced employing the dip-coating technique with increasing withdrawal speeds. However, with this procedure, the maximum thickness obtained is about two times the value of initial thickness. By contrast, partial vapor pressure gradient can promote much larger relative thickness variations. Moreover, exploiting the effect described in this work to produce 14 
controlled thickness gradient allows preparing graded films at a constant withdrawal speed, in a very simple process which doesn't require elaborate dip-coating equipment.

As it's highlighted in equation 4, the factor which has to be controlled is the relative solvent vapor pressure in the atmosphere $P_{a m b}$. If one target is building a linear film thickness profile, we are facing the fact that a linear evolution of $\Delta z$ caused by the substrate dragging induces at the solvent/air interface a $P_{a m b}(\Delta z)$ which has not a linear evolution inside the container. Hence, ideally one should find a way to promote a linear solvent relative pressure within the reservoir. This can be done by using a reservoir with inside walls distance widening from the bottom to the top. Although this approach is reliable, it is not very convenient, because a new container should be designed for every different solution composition and every temperature to obtain a chosen graded profile. In a more practical way, another simpler method consists in playing on the ratio between the substrate volume and the solution volume $\mathrm{V}_{\text {sample }} / \mathrm{V}_{l}$. The goal is to maintain the total solution lowering $\Delta z$ (promoted by substrate dragging) within the range in which the surrounding atmosphere is far from saturation (the first centimeter of $\Delta z$ in figure 5 experiments for example). In this $\Delta z$ range, the diffusion profile of $P_{a m b}$ can be considered as a reasonable linear approximation. This could lead to the preparation of graded layers exhibiting large thickness gradient along the specimen.

We tested this method ( $c f$. Figure 7) by varying the solvent to substrate volume ratio in order to vary the solution total lowering $\Delta z$. For a small volume ratio of 0.21 , resulting in a small $\Delta z$, the deposited layer has a thickness gradient which is perfectly linear but of moderate amplitude. A too high volume ratio of 0.43 does not allow getting a linear thickness gradient. With an intermediate volume ratio of 0.32 however, one obtains a regular thickness gradient decreasing from $74 \mathrm{~nm}$ to $16 \mathrm{~nm}$.

Thus, controlled thickness gradients can be obtained with a very simple constant speed dipcoating process by knowing and compensating the solvent vapor pressure variations around 15 
the meniscus. Remarkably, the thickness variation observed here (4.6 fold between smaller and larger thicknesses) is larger than that previously obtained by using accelerating dipcoating process $(2.5$ folds $) .^{25}$

\section{CONCLUSIONS}

We presented an experimental investigation of the impact of the variation of solvent relative pressure that takes place during dip-coating processing. This study highlighted that solvent relative pressure is responsible for the difficulties often met in literature for fitting experimental film thickness with Landau-Levich equation. A more accurate description of dip-coating deposition processing must take into account the evaporation speed of the solvent which varies a lot as a function of the solvent, the geometry of the solution reservoir, and the lowering of the solution level within this reservoir. We demonstrated that a good knowledge of these parameters allows for the controlled deposition of film thickness gradients of large amplitude.

\section{ASSOCIATED CONTENT}

\section{Supporting Information.}

The Supporting Information is available free of charge on the ACS Publications website at DOI:

Experimental points obtained for all the studied systems, effect of the shape and size of the container and of the difference in liquid meniscus shape, scheme of the apparatus used in dipcoating experiments with controlled relative solvent vapor pressure.

\section{AUTHOR INFORMATIONS}

16 


\section{Corresponding author}

*E-mail: cedric.boissiere@upmc.fr

\section{Notes}

The authors declare no competing financial interest

\section{AKNOWLEDGEMENTS}

We acknowledge the financial support from the DIM CNano Region Ile-de-France.

\section{ABBREVIATIONS}

LL Landau-Levich; CR Capillarity Regime

\section{REFERENCES}

1. Brinker, C. J., Hurd, A. J., Schunk, P. R., Frye, G. C. \& Ashley, C. S. Review of Sol-Gel Thin Film Formation. J Non-Cryst Solids 1992, 147, 424-436.

2. Brinker, C. J., Frye, G. C., Hurd, A. J. \& Ashley, C. S. Fundamentals of Sol-Gel Dip Coating. Thin Solid Films 1991, 201, 97-108.

3. Brinker, C. J., Lu, Y., Sellinger, A. \& Fan, H. Evaporation-Induced Self-Assembly: Nanostructures Made Easy. Adv. Mater. 1999, 11, 579-585.

4. Scriven, L. E. Physics and Applications of Dip-Coating and Spin Coating. in Symposium H - Better Ceramics Through Chemistry III 1988,121, 717-730. 
5. Takahashi, Y., Okada, S., Bel Hadj Tahar, R., Nakano, K., Ban, T. \& Ohya, Y. DipCoating of ITO Films. J. Non-Cryst. Solids 1997, 218, 129-134.

6. Jang, J., Nam, S., Im, K., Hur, J., Cha, S. N., Kim, J., Son, H. B., Suh, H., Loth, M. A., Anthony, J. E. et al. Highly Crystalline Soluble Acene Crystal Arrays for Organic Transistors: Mechanism of Crystal Growth During Dip-Coating. Adv. Funct. Mater. 2012, $22,1005-1014$.

7. Mirri, F., Ma, A. W. K., Hsu, T. T., Behabtu, N., Eichmann, S., Young, C. C., Tsentalovich, D. E. \& Pasquali, M. High-Performance Carbon Nanotube Transparent Conductive Films by Scalable Dip-Coating. ACS Nano 2012, 6, 9737-9744.

8. Valle, G. G., Hammer, P., Pulcinelli, S. H. \& Santilli, C. V. Transparent and Conductive ZnO:Al Thin Films Prepared by Sol-Gel Dip-Coating. J. Eur. Ceram. Soc. 2004, 24, 1009 1013.

9. Cathro, K., Constable, D. \& Solaga, T. Silica Low-Reflection Coatings for Collector Covers, by a Dip-Coating Process. Sol. Energy 1984, 32, 573-579.

10. Sonawane, R. ., Hegde, S. . \& Dongare, M. . Preparation of Titanium(IV) Oxide Thin Film Photocatalyst by Sol-Gel Dip-Coating. Mater. Chem. Phys. 2003, 77, 744-750.

11. Sonawane, R. ., Kale, B. . \& Dongare, M. . Preparation and Photo-Catalytic Activity of Fe/TiO2 Thin Films Prepared by Sol-Gel Dip-Coating. Mater. Chem. Phys. 2004, 85, $52-57$.

12. Lindqvist, K. \& Lidén, E. Preparation of Alumina Membranes by Tape Casting and Dip-Coating. J. Eur. Ceram. Soc. 1997, 17, 359-366.

13. Ge, C., Xie, C. \& Cai, S. Preparation and Gas-Sensing Properties of Ce-Doped ZnO Thin-Film Sensors by Dip-Coating. Mater. Sci. Eng. B 2007, 137, 53-58.

14. Shieh, J., Feng, H. M., Hon, M. H. \& Juang, H. Y. WO3 and W/Ti/O Thin-Film Gas Sensors Prepared by Sol-Gel Dip-Coating. Sens. Actuators B Chem. 2002, 86, 75-80. 
15. Landau, L. \& Levich, B. Dragging of a Liquid by a Moving Plate. Acta Physicochim. Urss 1942, 17, 42-54.

16. Faustini, M., Louis, B., Albouy, P. A., Kuemmel, M. \& Grosso, D. Preparation of Sol-Gel Films by Dip-Coating in Extreme Conditions. J. Phys. Chem. C 2010, 114, 76377645.

17. Kuznetsov, A. V. \& Xiong, M. Effect of Evaporation on Thin Film Deposition in Dip Coating Process. Int Commun Heat Mass Transf. 2002, 29, 35-44.

18. Lee, C. H., Lu, Y. F. \& Shen, A. Q. Evaporation Induced Self Assembly and Rheology Change During Sol-Gel Coating. Phys Fluids 2006, 18, 052105-052105-11.

19. Grosso, D. How to Exploit the Full Potential of the Dip-Coating Process to Better Control Film Formation. J Mater Chem 2011, 21, 17033-17038.

20. Sanchez, C., Boissiere, C., Grosso, D., Laberty, C. \& Nicole, L. Design, Synthesis, and Properties of Inorganic and Hybrid Thin Films Having Periodically Organized Nanoporosity. Chem.Mater. 2008, 20, 682-737.

21. Soler-Illia, G. J. A. A., Angelome, P. C., Fuertes, M. C., Grosso, D. \& Boissiere, C. Critical Aspects in the Production of Periodically Ordered Mesoporous Titania Thin Films. Nanoscale 2012, 4, 2549-2566.

22. O’Regan, B. \& Gratzel, M. A Low-Cost, High-Efficiency Solar Cell Based on DyeSensitized Colloidal TiO2 Films. Nature 1991, 353, 737-40.

23. Sakatani, Y. et al. Optimised Photocatalytic Activity of Grid-Like Mesoporous TiO2 Films: Effect of Crystallinity, Pore Size Distribution, and Pore Accessibility. J Mater Chem 2006, $16(1), 77-82$.

24. Bosca, F., Edwardsa, D., Kellera, N., Kellera, V. \& Ayral, A. Mesoporous TiO2-based Photocatalysts for UV and Visible Light Gas-Phase Toluene Degradation. Thin Solid Films 2006, 495 (1-2), 272-279. 
25. Louis, B., Krins, N., Faustini, M. \& Grosso, D. Understanding Crystallization of Anatase into Binary $\mathrm{SiO}_{2} / \mathrm{TiO}_{2}$ Sol-Gel Optical Thin Films: An in Situ Thermal Ellipsometry Analysis. J. Phys. Chem. C 2011, 115, 3115-3122.

26. John Biteau, Christy Ford, Arnaud Glacet. Method of Dip-Coating a Lens, US20130022739 A1 2013

27. Hertz, H. On the Evaporation of Liquids, Especially Mercury. In Vacuo. Ann Phys Chem 1882, 17, 177-198.

28. Knudsen, M. Die maximale Verdampfungsgeschwindigkeit des Quecksilbers. Ann. Phys. 1915, 352, 697-708.

29. Ceratti, D. R., Louis, B., Paquez, X., Faustini, M. \& Grosso, D. A New Dip Coating Method to Obtain Large-Surface Coatings with a Minimum of Solution. Adv. Mater. 2015, $27,4958-4962$.

30. Faustini, M. et al. Engineering Functionality Gradients by Dip Coating Process in Acceleration Mode. ACS Appl. Mater. Interfaces 2014, 6, 17102-17110. 
a)

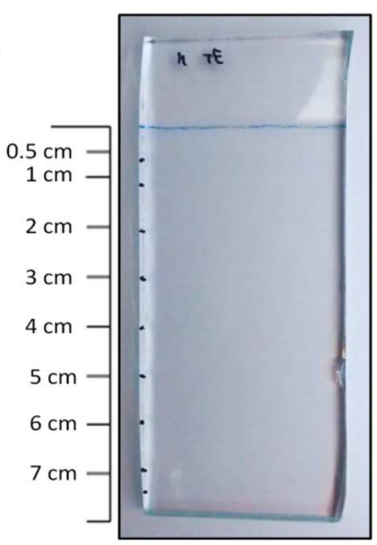

b)

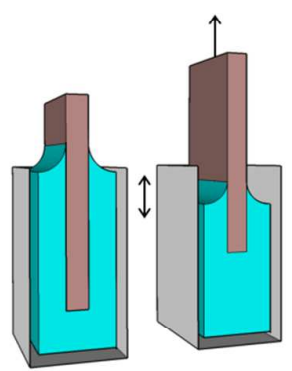

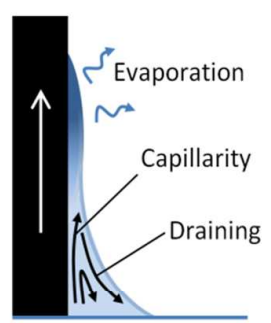

Draining regime

Figure 1: Scheme of capillarity and draining regimes which dominate the dip-coating process respectively at slow and fast withdrawal speeds. Capillarity regime can be very useful in case of aqueous solutions because it avoids dewetting problems; moreover it enables us to deposit thick films from highly diluted solutions.

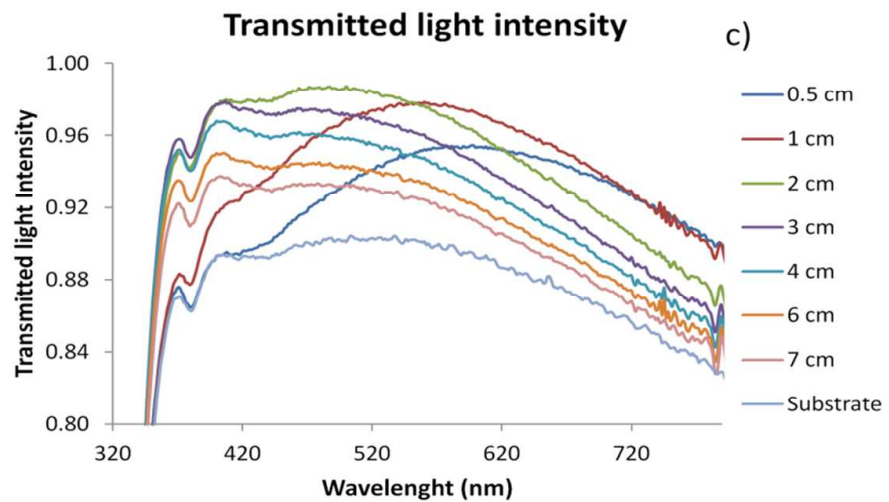

d)

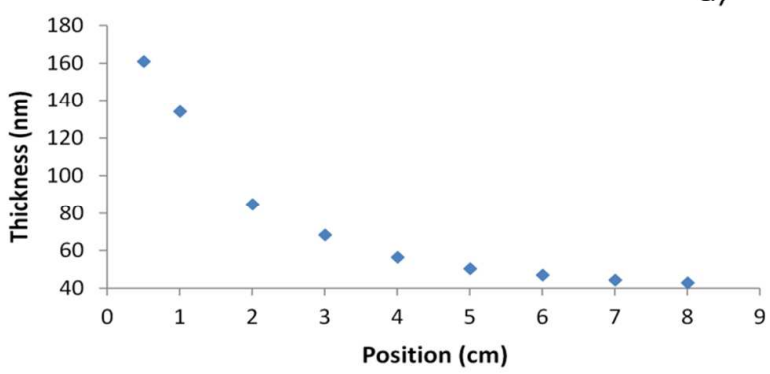

Figure 2: a) Glass substrate coated with a single anti-reflective layer, various positions on the sample for which transmission and layer thickness have been measured are reported b) Dip-coating conditions: the liquid level inside the container decreases during the process because of the substrate dragging. Withdrawal speed used: 0.3 $\mathrm{mm} \mathrm{s}^{-1}$, c) Transmission spectra taken at different distances from the top edge of the coating layer d) Thickness profile of the sample. 
Figure 3: Scheme of the dip-coating method and plot of the thickness versus withdrawal speed (log-log scale) when the dip-coating is performed with different reservoirs (curves A,B and C) or same reservoir but different level of liquid (curves $\mathrm{C} 1, \mathrm{C} 2, \mathrm{C} 3$ ) for a $\mathrm{TiO}_{2}$ solution in EtOH. The reported graphs are the fits obtained from experimental data points following the equation 1 . The data points were acquired at $0.5 \mathrm{~cm}$ from the top of the film. 

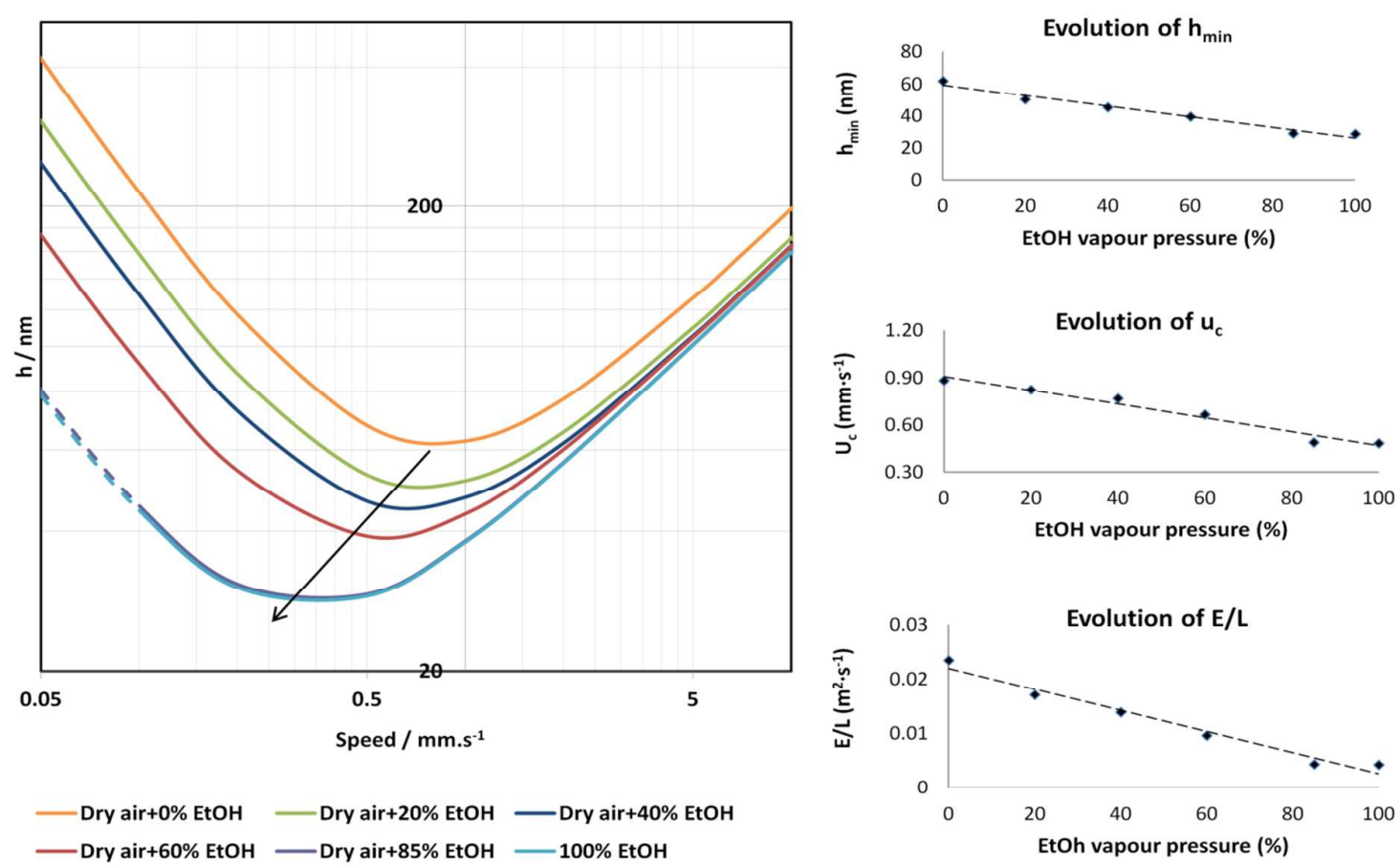

Figure 4: Plot of the thickness versus withdrawal speed (log-log scale) for different solvent vapor pressures injected in the dip-coater chamber (experimental points are given in SI1, the data points were acquired at $0.5 \mathrm{~cm}$ from the top of the film.). Dashed lines are the theoretical trends, which are not possible to verify because dewetting from substrate surface occurs at speeds smaller than $0.1 \mathrm{~mm} \mathrm{~s}^{-1}$ for high values of EtOH vapor pressure. To have a clear image we report here just the fitted curves, the experimental points are presented in the supplementary information. On the right are shown evolutions of the minimal thickness $h_{\min }$, the critical withdrawal speed $u_{c}$ (at which $h_{\min }$ is obtained), and the solution deposition rate E/L (from Equation 1) with solvent vapor pressure injected into the dip-coater chamber. 
Mesoporous $\mathrm{SiO}_{2}$ in $\mathrm{EtOH}$
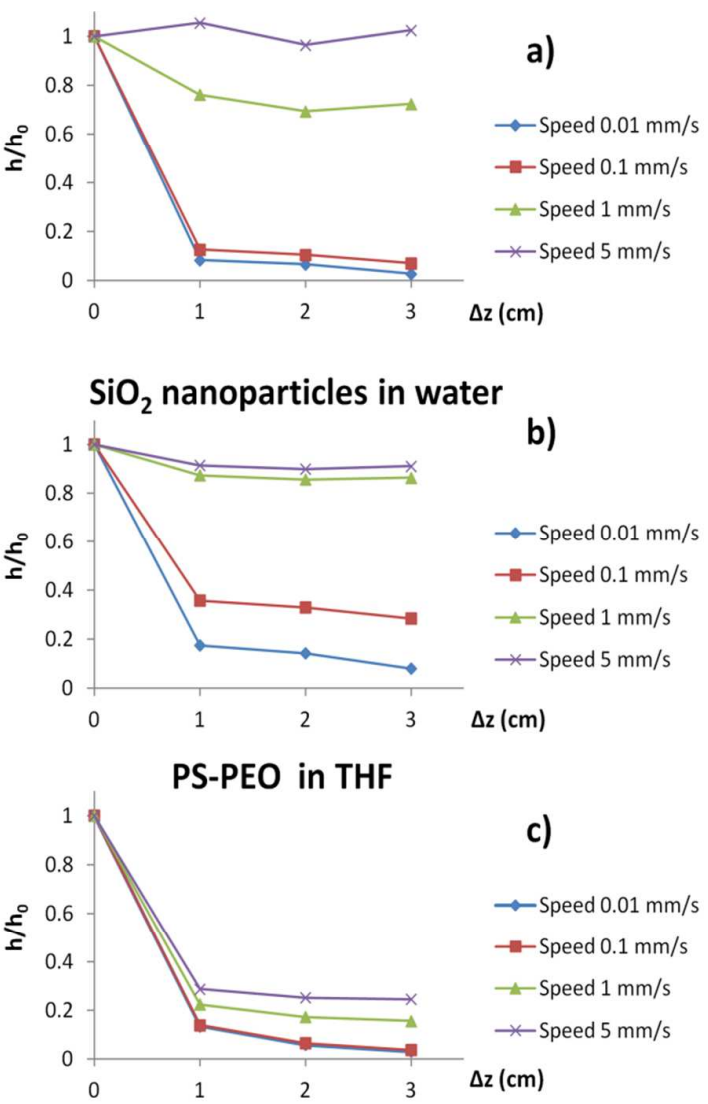

Figure 5: : Evolution of $\mathrm{h} / \mathrm{h}_{0}$ with the difference in liquid level $\Delta \mathrm{z}$ for different speeds, for: a) ethanol-based sol-gel $\mathrm{SiO}_{2}$ solution b) water-based colloidal $\mathrm{SiO}_{2}$ solution, c) THF-based PS-PEO block copolymer solution. $\mathrm{The} h / \mathrm{h}_{0}$ is the ratio between the thickness of a film dip-coated with a liquid level within the reservoir lowered by $1,2,3 \mathrm{~cm}$ and a sample dip-coated at the same speed but with the liquid level reaching the top edge of the container. Complete thickness versus withdrawal speed data are provided in SI 2 to 4. 
a)
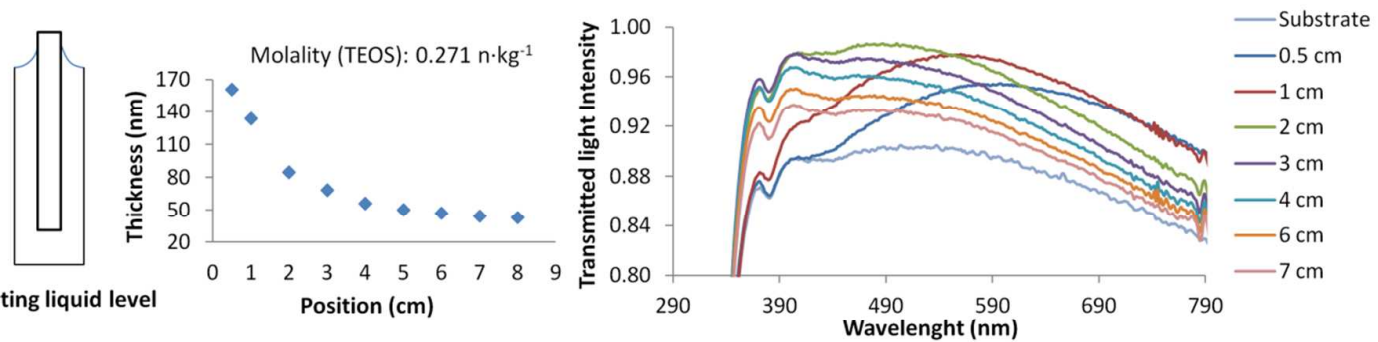

b)

Starting liquid level

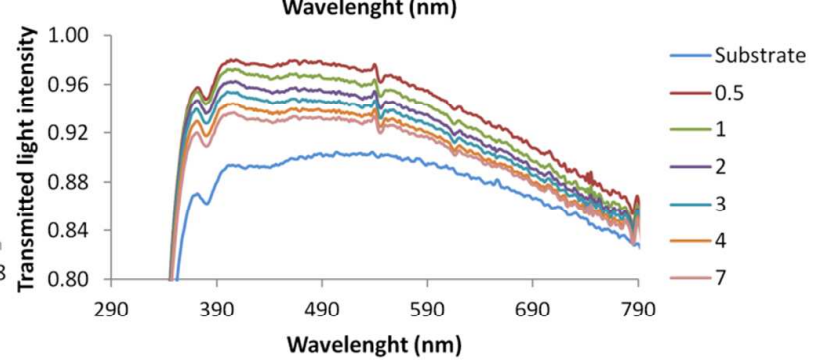

Starting liquid level

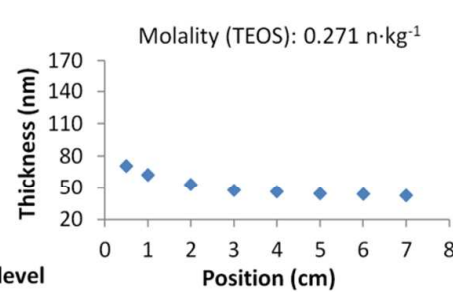

c)
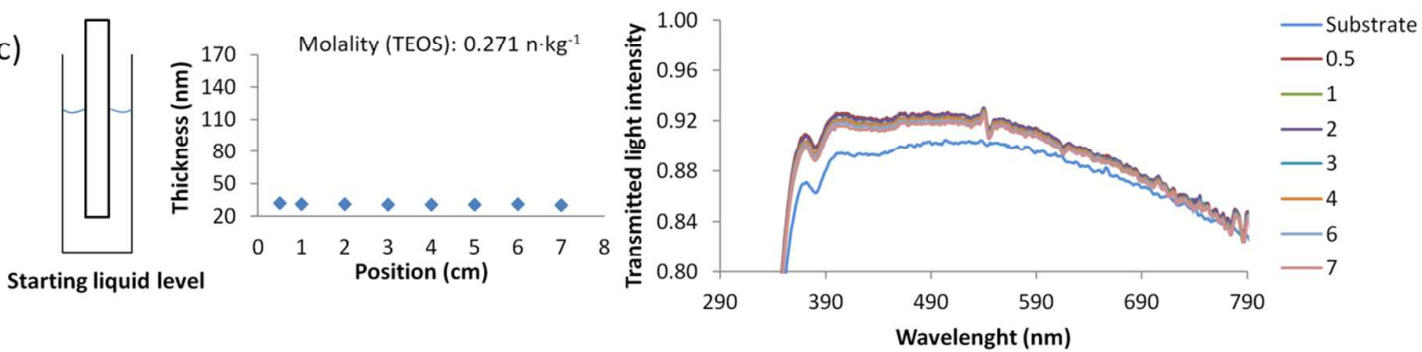

d)
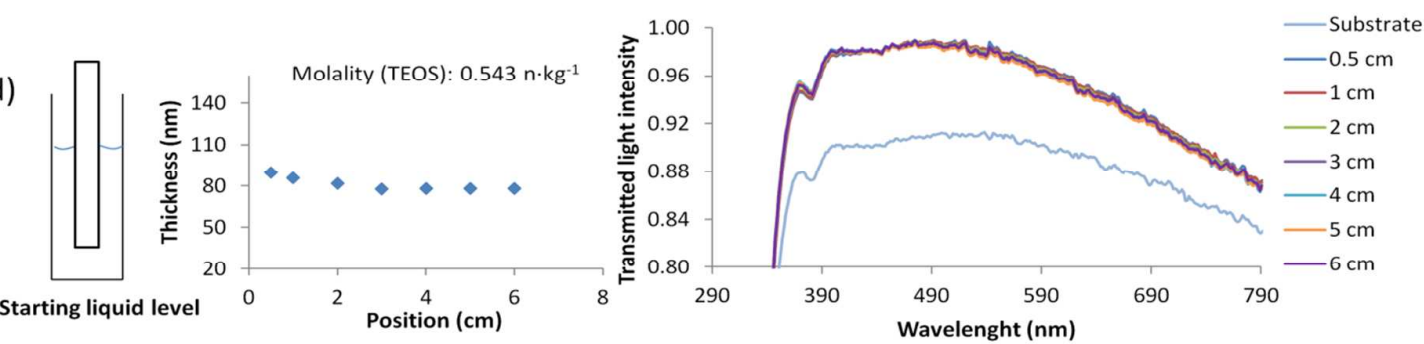

Figure 6: Dip-coating of an anti-reflective layer on a sodalime glass dip-coated at $0.3 \mathrm{~mm} \mathrm{~s}^{-1}$. (left) film thickness profile obtained at different positions of the substrate, (right) UV-Vis Transmission spectra at similar positions of the substrates. The films are obtained by dip-coating a mesoporous silica sol-gel solution (TEOS/MTEOS 0.5:0.5), compositions are given in Experimental section. From a) to c) are shown that lowering the liquid level within the container leads to more uniform films deposition, but the layer becomes too thin to have good anti-reflective properties with the original solution. d) By readapting the solution concentration and dip-coating with the meniscus inside the reservoir, we obtain quasi-homogeneous film thickness and good antireflective properties along the whole sample. 
Volume ratio: 0.21

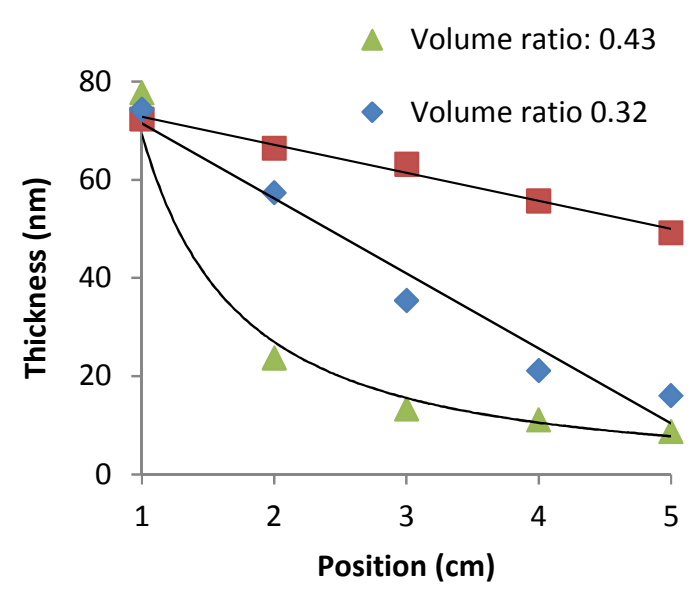

Figure 7: Plot of the thickness of graded mesoporous $\mathrm{SiO}_{2}$ films for different volume ratio between the sample and the solution. The ethanol-based solution was dip-coated at withdrawal speed $0.1 \mathrm{~mm} \mathrm{~s}^{-1}$. Black lines are given as guide for the eyes. 


\section{TOC graphic:}

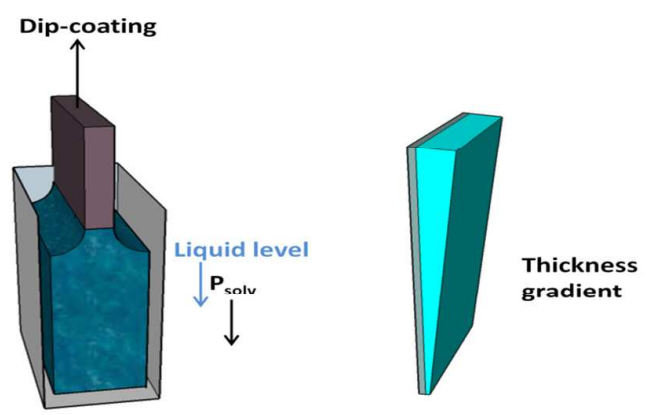

